\title{
Safety and feasibility of prehospital thrombolysis carried out by paramedics
}

Phil Keeling, Debbie Hughes, Linnie Price, Steve Shaw, Andy Barton

The benefits of thrombolysis in patients with acute myocardial infarction are time dependent, with a potential $48 \%$ reduction in mortality if treatment is received within an hour of onset of symptoms. ${ }^{1}$ The benefits diminish thereafter. However the "call to needle" standard set by the national service framework ${ }^{2}$ has been achieved for only a third of patients nationally because of lengthy ambulance journeys and delays in hospital. Prehospital thrombolysis may be the most feasible means of achieving timely reperfusion, and all published studies have shown the accuracy of diagnosis, patient selection, and administration of thrombolysis. ${ }^{3}$ The Joint Royal Colleges Ambulance Liaison Committee favours autonomous prehospital thrombolysis by paramedics as a long term objective. ${ }^{4}$ We tested the feasibility of physician assisted and autonomous models of paramedic prehospital thrombolysis.

\section{Methods and results}

All 64 paramedics serving the study hospital took part in the study. Of these, $48(75 \%)$ received formal training in recording 12 lead electrocardiograms, diagnosing ST elevation acute myocardial infarction (STEMI), and assessing suitability for thrombolysis with a checklist of nationally agreed exclusion and inclusion criteria. Those who were not formally trained acquired the necessary skills from trained paramedics via a cascade system. During one year electrocardiograms of patients with category A (emergency) chest pain were transmitted to hospital by telemetry and reviewed by the duty emergency medicine physician. Paramedics recorded data on the diagnoses of physicians and paramedics, the paramedics' hypothetical decisions to treat, and timings of all events, although they did not actually administer the drug. The actual time to thrombolysis and a consultant's diagnosis from the transmitted electrocardiogram was used as the standard. We analysed data for sensitivity and specificity of diagnoses and potential reduction in call to needle times. Where appropriate we calculated 95\% confidence intervals.

Of 1192 emergency admissions for chest pain, we had complete data for $861(72 \%)$ regarding the paramedics' diagnosis of STEMI. We consider this sample to be representative because failures in data collection were due to random incidence of transmission and equipment and protocol failure. The number of "true" STEMIs (according to the consultant's diagnosis) was 118 (14\%). Among the 660 cases for which both the paramedic's and the physician's diagnosis was available, the paramedics' sensitivity was $71 \%(84 / 118)$ and specificity 97\%. McNemar's test showed that paramedics' sensitivity was lower than that of doctors $(71 \% v 90 \%, \mathrm{P}=0.001)$ but the specificity was higher $(97 \%$ ข $94 \%, \mathrm{P}=0.001)$.

The median call to potential needle time (call for help to paramedic's decision) was 28 minutes (range 7-112 minutes), and for STEMI the median time saved was 48 minutes. The goal of call to needle time in less than 60 minutes was met in $95 \%$ of cases, and $22 \%$ of patients would have received thrombolysis within 60 minutes of onset of symptoms. At present only $3 \%$ do so. The table shows the summary statistics of times measured. We have included under each category all cases for which we have appropriate data but the inconsistencies of some databases and transmission and protocol failures have led to differing denominators.

\section{Comment}

This feasibility study of autonomous prehospital thrombolysis shows that paramedics can record and interpret 12 lead electrocardiograms and safely administer thrombolysis in the community. The potential average time saved was 48 minutes from the call for help to medication being administered. The physician assisted model was found to be unreliable because of technological failure and areas in which communication was hampered because mobile phones could not work, a problem observed elsewhere. ${ }^{5}$ We did not routinely collect data for these unanticipated events so their precise distribution cannot be reported. Autonomous paramedic prehospital thrombolysis seemed feasible and safe and was associated with improved call to needle times. Sensitivity may improve with experience and confidence.

Summary statistics for time from event to response (minutes)

\begin{tabular}{|c|c|c|c|c|}
\hline \multirow[b]{2}{*}{ Event-response } & \multicolumn{2}{|c|}{ All events } & \multicolumn{2}{|c|}{ STEMI only } \\
\hline & No of cases & Median (range) & No of cases & Median (range) \\
\hline Onset of symptoms-call for help & 189 & $84(0-1285)$ & 88 & $74(0-1285)$ \\
\hline Onset of symptoms-paramedic's decision & 198 & $120(9-250)$ & 82 & $110(16-1250)$ \\
\hline Onset of symptoms-needle & 106 & $188(50-1428)$ & 82 & $172(50-1330)$ \\
\hline Call for help-arrival of ambulance & 196 & $10(1-98)$ & 88 & $21(3-98)$ \\
\hline Call for help-paramedic's decision & 166 & $28(7-112)$ & 75 & $29(10-100)$ \\
\hline Call for help-arrival at hospital & 199 & $51(9-147)$ & 90 & $54(9-130)$ \\
\hline Call for help-needle & 101 & $85(33-1236)$ & 77 & $75(33-780)$ \\
\hline Paramedic's decision-physician assisted decision & 576 & $5(0-67)$ & 78 & $5(0-25)$ \\
\hline Paramedic's decision-actual thrombolysis (time saved) & 101 & $57(18-1208)$ & 72 & $48(18-813)$ \\
\hline Hospital door-needle & 109 & $26(2-1189)$ & 83 & $21(2-650)$ \\
\hline
\end{tabular}

STEMI=ST elevation acute myocardial infarction.

South Devon

Healthcare Trust, Lawes Bridge, Torquay TQ2 7AA Phil Keeling consultant cardiologist Debbie Hughes clinical nurse specialist

Research and Development Support Unit, Peninsula Medical School, Plymouth PL6 8BX

Linnie Price research coordinator Andy Barton RDSU coordinator

Department of Mathematics and Statistics, University of Plymouth,

Plymouth PL4 8AA

Steve Shaw senior lecturer in statistics

Correspondence to: P Keeling phil.keeling@ nhs.net 
We thank the paramedics who took part in the study. The study was supported by a grant from NHSE South West Research and Development Directorate.

Contributors: PK was the principal investigator and was responsible for project design, data collection, advice on analysis, delivery of training, and drafting the paper. $\mathrm{DH}$ was responsible for project design, data collection, liaison with paramedics, delivery of training, and drafting the paper. LP was responsible for data collection, liaison with accident and emergency departments and paramedics, and drafting the report and paper. SS was responsible for project design, monitoring and analysis of data, and drafting the paper. $\mathrm{AB}$ was responsible for project design and management, advice on analysis, and drafting the paper and is guarantor for the study. Helen Myers and Dougie Williams (Westcountry Ambulance Service) and Paul Feasby (South Devon Healthcare Trust) ensured protocol compliance; Terry Phillips (Westcountry Ambulance Service) was responsible for delivery of training; and Margaret Somerville (Teignbridge PCT) and Ken Wenman (Westcountry Ambulance Service) advised on protocol.
Funding: NHS Executive Research and Development directorate South West. The guarantor accepts full responsibility for the conduct of the study, had access to the data, and controlled the decision to publish.

Competing interests: None declared.

Boersma E, Maas ACP, Dekkers JW, Simoons MI. Early thrombolytic treatment in acute myocardial infarction: reappraisal of the Golden Hour. Lancet 1996;348:771-5.

2 Department of Health. National Service Framework for coronary heart disease: standards five to seven: heart attack, acute myocardial infarction and other acute coronary syndromes. www.nelh.nhs.uk/nsf/chd/nsf/ main/main std5_6_7.htm (accessed 17 Sep 2001).

3 Morrison LJ, Verbeek PR, McDonald AC, Sawadsky BV, Cook DJ. Mortality and prehospital thrombolysis for acute myocardial infarction. A meta-analysis. JAMA 2000:31:2686-92.

4 Royal Colleges Ambulance Liaison Committee/Ambulance Services Association. Pre-hospital thrombolysis. London: National Institute for Clinical Excellence, 2001. (Technology appraisals process series 4)

5 National Institute for Clinical Excellence. Guidance on the use of drugs for early thrombolysis in the treatment of acute myocardial infarction. London: NICE, 2002. (Technology appraisal guidance No 52)

(Accepted 23 June 2003)

\title{
Abstinence from smoking eight years after participation in randomised controlled trial of nicotine patch
}

\author{
Patricia Yudkin, Kate Hey, Sarah Roberts, Sarah Welch, Michael Murphy, Robert Walton
}

Department of Primary Health Care, University of Oxford, Institute of Health Sciences, Oxford OX3 7LF

Patricia Yudkin reader in medical statistics

Cancer Research UK General Practice Research Group, Institute of Health Sciences, Oxford OX3 7LF

Kate Hey research officer

Michael Murphy director

Sarah Roberts research nurse

Sarah Welch research nurse

Department of Clinical

Pharmacology,

University of

Oxford, Radcliffe

Infirmary, Oxford

OX2 6HE

Robert Walton

senior research fellow

Correspondence to:

P Yudkin

pat.yudkin@

dphpc.ox.ac.uk

BMJ 2003;327:28-9
Few studies have investigated abstinence beyond three years among participants who stop smoking during trials of nicotine replacement therapy, ${ }^{1-3}$ and even fewer have followed up smokers who failed to quit during such trials. We carried out an eight year follow up of people who had participated in a randomised controlled trial of the nicotine patch.

\section{Participants, methods, and results}

Participants were the 1686 patients from general practices in Oxfordshire who took part in a double blind randomised controlled trial of the patch in 1991-2.. ${ }^{4}$ At entry they smoked $\geq 15$ cigarettes a day and were aged 25-64 years. Participants wore the patches for 12 weeks. The main outcome was abstinence from smoking for one year, confirmed at 12, 24, and 52 weeks by a salivary cotinine concentration $\leq 20 \mathrm{ng} / \mathrm{ml}(89 \%$ of cases) or expired carbon monoxide $\leq 10 \mathrm{ppm}(11 \%)$.

In 1999-2000, we contacted 1532 of the 1625 living participants. We sent two follow up letters and phoned non-responders. In total 840 participants completed a questionnaire giving demographic details and information about smoking. The mean time from enrolment in the trial to follow up was 8.3 (SD 0.35) years, with a range of 7.4-9.3 years. Responders were more likely to be women $(59.0 \%$ v $51.7 \% ; \mathrm{P}=0.005)$ and were more likely to have stopped smoking during the trial than non-responders $(13.2 \% v 5.5 \%$ quit for one year; $\mathrm{P}<0.0001)$. Reported abstinence at follow up was confirmed by a plasma cotinine concentration $\leq 20$ $\mathrm{ng} / \mathrm{ml}$. Responders reported for how long they had been abstinent. We assumed that all those lost to follow up were still smoking.

Of the 153 participants who had stopped smoking for a year in the original trial, 83 were still not smoking at follow up, giving an eight year abstinence rate of
$83 / 1625$ (5\%; $95 \%$ confidence interval $4 \%$ to $6 \%)$ and a relapse rate of $70 / 153(46 \% ; 38 \%$ to $54 \%)$ (table). Relapse was similar in active and placebo groups: the active/placebo odds ratio (OR) for continuous abstinence up to follow up was 1.39 (0.89 to 2.17; $\mathrm{P}=0.19$ ) compared with 1.45 (1.04 to $2.03 ; \mathrm{P}=0.03$ ) for quitting for a year in the trial.

Of the 1472 who did not quit for a year in the trial, $116(8 \% ; 7 \%$ to $9 \%)$ were abstinent at follow up. Of these, $89(6 \% ; 5 \%$ to $7 \%)$ had abstained for a year or more, and 27 for less than a year (median 4 months). Overall at follow up therefore, $172(11 \% ; 9 \%$ to $12 \%)$ of trial participants had been abstinent for a year or more, $29(2 \%)$ had been abstinent for less than a year, and $1424(88 \%)$ were smoking.

\section{Comment}

Eight years after taking part in a randomised trial of the nicotine patch, just under half of the $9 \%$ who had stopped smoking for a year had relapsed, leaving $5 \%$ of all trial participants continuously abstinent for eight years. Previous studies have reported that a third to a half of all those who stop during a trial relapse by three or four years. ${ }^{1-3}$ Use of the nicotine patch conferred a $39 \%$ increase in the odds of continuous abstinence compared with placebo. The increase was not significant, but our original trial was not powered to detect the small difference observed in eight year abstinence rates $(5.9 \% v 4.3 \%)$. Of the majority who did not quit in the trial, only $8 \%$ had given up smoking at follow up, leaving $88 \%$ of trial participants still smoking. Our estimates were based on the conservative but well accepted assumption that those lost to follow up were still smoking. Finding more effective ways to help people to give up smoking remains an ongoing challenge. 